August 12-15, 2012, Chicago, IL, USA

DETC2012-70965

\title{
EXPLORATION OF MOBILITY MODES ARISING FROM THE BREAKDOWN OF A BASIC REGULAR TETRAHEDRON
}

\author{
Yin M. Chen \\ Department of Mechanical Engineering \\ Stevens Institute of Technology \\ Hoboken, New Jersey 07030 \\ Email: ychen1@stevens.edu
}

\author{
David Cappelleri \\ Department of Mechanical Engineering \\ Stevens Institute of Technology \\ Hoboken, New Jersey 07030 \\ Email: David.Cappelleri@stevens.edu
}

\begin{abstract}
One of the most important considerations in the design of robots is mobility. How does the system traverse the terrain and environment where it is expected to operate? In past efforts, engineers and scientists have received inspiration from man-in-the-loop vehicles and modes of mobility used by animals. While amazing advancements have been achieved, this top-down approach tends to focus on one specific solution to a problem which may cause other solutions to be overlooked. This paper approaches the mobility problem from a different point of view. Starting with the most basic three dimensional shape, a regular tetrahedron, we explore seven different modes of mobility arising from simple modifications of the initial shape. These resultant modes are then resolved into corresponding, existing mechanisms that are already widely applied. By using this bottom-up approach, we are able to explore available mobility modes in a much more comprehensive manner. Instead of starting with a complex system in mind, engineers will be able to use simple building blocks to enable different desired mobility behaviors.
\end{abstract}

\section{INTRODUCTION}

Robotics is a field of study that has been around since the 1700 s when marionettes were first programmed to play music [1]. As such, the word robot conveys a certain meaning or preconception. We think of human-like systems such Hondas Asimo, vehicular systems like the Mars Rover, as well as stationary, industrial robots that are arms with many linkages.

Throughout the history of the field, a great deal of consideration has been given to the study of different modes of mobility [2-21]. One of the main purposes of robots is to act as a hu- man proxy in environments where we cannot survive or perform as well as we would like. Be it interplanetary exploration [22], oceanography [23], or disaster search-and-rescue [24], there are limitations machines can overcome that are beyond the reach of humans. Hence, it is not surprising much of the inspiration for the different schema and types of mobility mechanisms comes from man-in-the-loop vehicles and mimicry of the movement of humans and other animals. The commonality between these design methods is they are all top-down approaches that start with a view or a goal of the behavior of the overall system.

While such methods are extremely useful for solving specific problems with an end goal in mind, it can obfuscate other options for solving the same problem. As with any other project, it is often beneficial to examine the problem from a different point of view. In this paper, we will approach the mobility problem from the bottom up. We will exercise the movement capabilities of the most basic of 3-dimensional shapes, a regular tetrahedron. For each mobility mode, we will examine the range of motion that is exhibited. Where applicable, we will also explore the different applications where it can be and is currently used.

\section{ASSUMPTIONS AND BOUNDARY CONDITIONS}

Let us start by defining our tetrahedron. A regular tetrahedron has 4 faces, 4 vertices, and 6 sides. All 4 faces are identical and all 6 sides have the same length. Since the deformation of lines is more easily defined than the deformation of planes, we will only consider the case where the tetrahedron is made up entirely of edges, a wire-frame structure with no faces. For the purpose of this study, we have chosen to consider the tetrahedron to only have 4 point masses at the vertices, so the location of 
the center of mass (CoM) will be considered the position of the tetrahedron (Fig. 1).

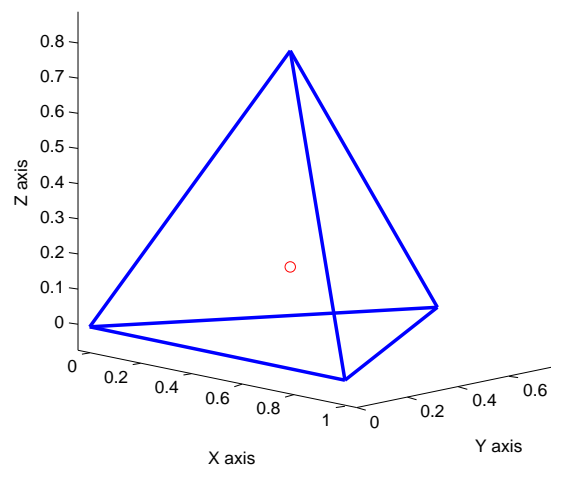

FIGURE 1: REGULAR TETRAHEDRON. THE CIRCLE REPRESENTS THE COM

It is trivial to see that this initial shape is fully constrained. Without an outside force, the tetrahedron CoM will not move. It is the goal of this paper to systematically remove the initial constraints in order to give the body mobility. In application, there will be many different ways of implementing each mode of mobility. Therefore, our calculations will be theoretical and ideal, without taking into account the possible masses and moments that will be required to actuate the motions.

It is also important to note that as we remove "key" constraints, secondary movements have to be allowed in order to take advantage of the new degrees of freedom. Therefore, we will assume that all edges are allowed free rotation relative to each other. There are no rotational constraints, only prismatic ones. In addition, all calculations are based on the assumption that this is a unit tetrahedron. In other words, each edge is of length 1 .

\section{MOBILITY MODE 1: PRISMATIC DOF}

\section{Submode 1A}

First, let us define a few reference points on the body as shown in Fig. 2a. The unit tetrahedron is made up of points A, B, $\mathrm{C}$ and $\mathrm{D}$ at the locations indicated. The first mode of mobility is achieved by removing a constraint from one end of a member and allowing it to become a prismatic joint. In this case we will use edge $\mathrm{CD}$ and allow the end touching point $\mathrm{D}$ to be the prismatic joint. Vertices $C$ and $D$ can now move closer together.

Figure 3 shows the range of motion that is allowed based on this degree of freedom. In this submode, the range of motion of the centroid is limited and may not be very useful. It can, however, be actuated three different ways: controlling the length of the edge that lies between the two vertices $(l)$, controlling the angle between the two remaining constrained triangular frames $\left(\theta_{1}\right)$, or controlling the angle of the loose edge at the base vertex $\left(\theta_{2}\right)$, as illustrated in Fig. $2 \mathrm{~b}$.

The resultant range of motion of the CoM as a function of each of the control variables is shown in Eqn. (1-3).

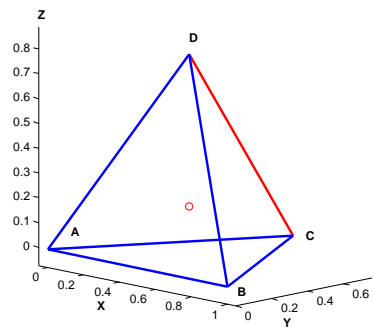

(a)

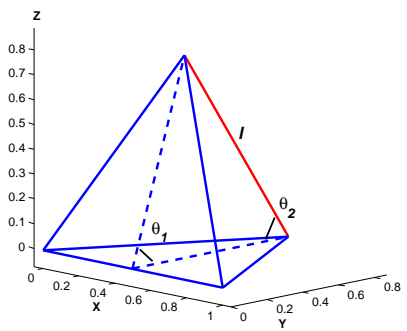

(b)
FIGURE 2: (a)A UNIT TETRAHEDRON WHERE THE VERTICES ARE AS FOLLOWS: A $(0,0,0), \mathrm{B}(1,0,0), \mathrm{C}(0.5,0.866,0), \mathrm{D}(0.5,0.289,0.816)$ (b)THE PARAMETERS THAT CAN BE USED TO CONTROL THE MOVEMENT

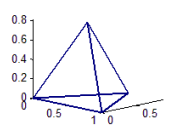

$\mathrm{t}=0$

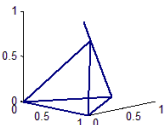

$\mathrm{t}=1$

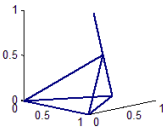

$\mathrm{t}=2$

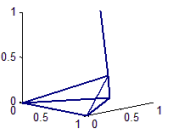

$t=3$
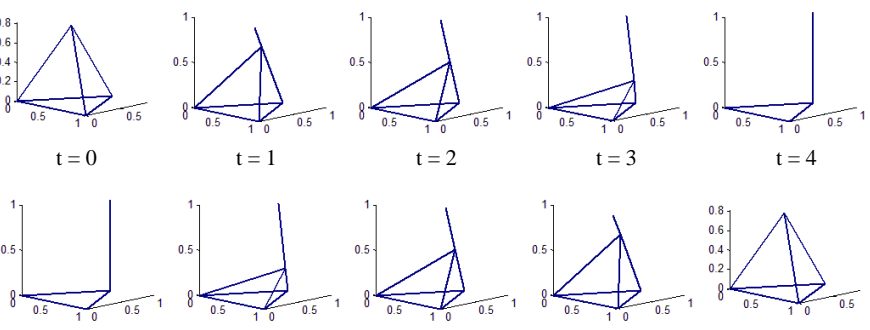

$\mathrm{t}=5$

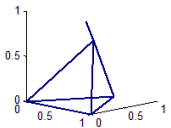

$\mathrm{t}=7$

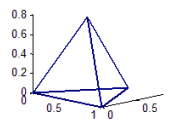

$\mathrm{t}=8$
FIGURE 3: THE MOVEMENT OF THE TETRAHEDRON BODY AS IT ACTUATES ALONG THE "FREED" EDGE

For $l=[0,1]$

$$
C M_{l}=\left[\frac{1}{2}, \frac{\sqrt{3}}{4}\left(1-\frac{l^{2}}{3}\right), \frac{1}{4} \sqrt{l^{2}-\frac{1}{3} l^{4}}\right]^{T}
$$

For $\theta_{1}=[0,1.231]$ radians

$$
C M_{\theta_{1}}=\left[\frac{1}{2}, \frac{\sqrt{3}}{8}\left(1+\cos \theta_{1}\right), \frac{\sqrt{3}}{8} \sin \theta_{1}\right]^{T}
$$

For $\theta_{2}=[0.955,1.571]$ radians

$$
C M_{\theta_{2}}=\left[\frac{1}{2}, \frac{\sqrt{3}}{4}\left(1-\cos ^{2} \theta_{2}\right), \frac{\sqrt{3}}{4} \sin \theta_{2} \cos \theta_{2}\right]^{T}
$$

As one can see, in order to actuate the body from one extreme to the other, we must go from $l=0$ to $l=1$. This corresponds to 1.231 radians of motion on $\theta_{1}$ but only 0.616 radians on $\theta_{2}$. This makes $\theta_{2}$ the more efficient control point but the motor used here for actuation must be more robust in order to compensate for the larger resultant torque. In terms of the final range of motion of the centroid, the $\mathrm{x}$-component stays in the $\mathrm{x}=0.5$ plane. The $\mathrm{y}$ - and $\mathrm{z}$-components move slightly as shown in Fig. 4. From the calculations, the entire range of the CoM is merely 0.25 or a quarter of the length of an edge. In this situation, while there is a slight shift in location, it would be a stretch to call the body mobile by any conventional definition. The CoM does not move enough to cause tumbling and the tetrahedron is merely "flexing" in place. If we change the submode of the tetrahedron, however, we may indeed achieve mobility. 

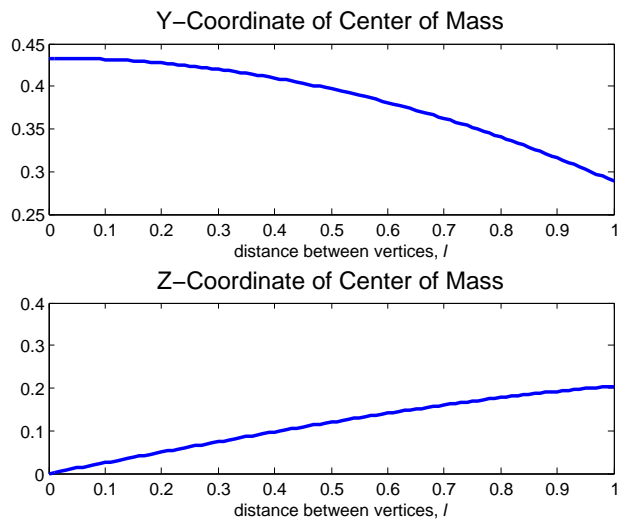

FIGURE 4: SUBMODE 1A: Y- AND Z- COMPONENTS OF COM MOTION IN TERMS OF $l$

For all subsequent modes of mobility, we can assume at least the same three relative actuation points can be used to change the distance between any two vertices of the tetrahedron. Only the most interesting actuation scheme will be discussed for each mode in the remainder of the paper. In addition, the comprehensive set of equations can be found in the technical report accompanying this paper [25].

\section{Submode 1B}

Starting with the same orientation as in Fig. 2, we will now actuate the distance between two of the base vertices instead. In the application of this mode of mobility, we must be sure to account for the contact points in order to ensure that the motion achieved is directional. Otherwise, we would be oscillating in place once again. A great deal of work has been done on the subject of mechanical contacts, including robotic feet [26-28]. It will be up to the engineer to select the appropriate ones for a given application.

In this case, let us make the assumption that vertex A will remain stationary while vertex $B$ moves toward vertex $C$. Both vertex $\mathrm{B}$ and vertex $\mathrm{C}$ are unidirectional contacts and can only move in the counter-clockwise direction around the z-axis. In this submode, we are also introducing the concept of gait. The subsequent movement will occur in two parts. In part 1, vertex B moves toward vertex $\mathrm{C}$, resulting in a collapsed triangle (Fig. $5 \mathrm{~b}$ top row). In part 2 , vertex $\mathrm{C}$ moves back away from vertex $\mathrm{B}$, finally arriving at the same relative vertices orientation as when we started, albeit with a resultant translation and rotation (Fig. 5b bottom row). In order to simplify the equations for this actuation, we will use a projection of the aforementioned $\theta_{1}$ on the X-Y plane as our control point (Fig. 5a). The resultant motion of the CoM is shown in Fig. 6. The governing equations can be found in the Technical Report. In application, this mechanism is similar to a disk cam with an out-of-plane displacement. The total travel of the CoM is 0.66 edge lengths during one gait cycle.

\section{Submode 1C}

Depending on the implementation, it is also possible to achieve linear motion with this mode of mobility. In Submode $1 \mathrm{~B}$, vertex A's contact with the "ground" is what keeps the tetrahedron moving in a circle. If only two points are in contact with
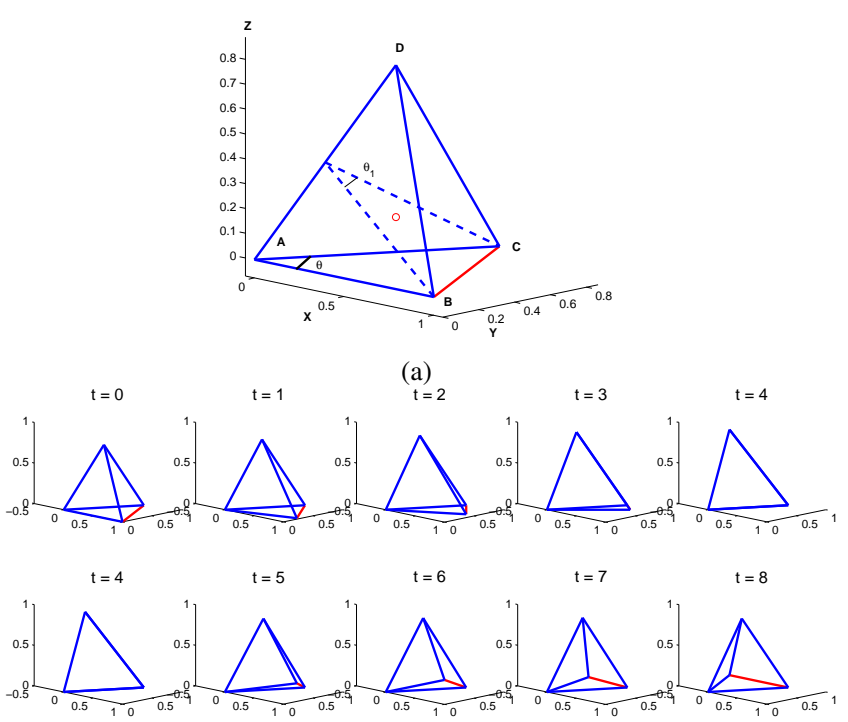

(b)

FIGURE 5: (a)THE ACTUATION POINT OF SUBMODE 1B. ANGLE $\theta_{1}$ IS PROJECTED ONTO THE X-Y PLANE AS $\theta$ FOR SIMPLICITY (b) THE MOTION OF THE TETRAHEDRON BODY AS IT ACTUATES THE EDGE BC

$\mathrm{X}$-Coordinate of Center of Mass
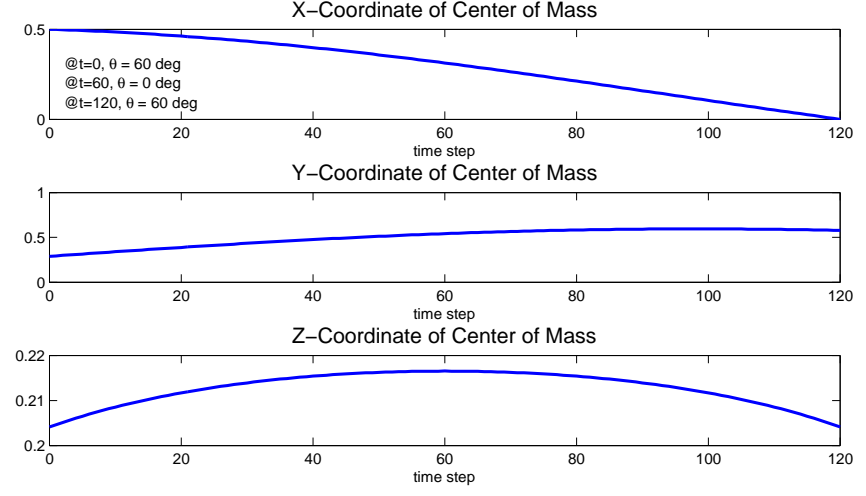

FIGURE 6: SUBMODE 1B:THE RESULTANT MOVEMENT OF THE COM

the "ground," and the distance between them is actuated, it would be possible for the tetrahedron to inch along in a linear fashion.

Submode $1 \mathrm{C}$ is defined as per Fig. 7a. Although the tetrahedron, as is, is not statically stable, let us assume this is an ideal case where the body can and does balance in this configuration. As mentioned previously, the exercise undertaken here is ideal and should any particular mechanism or mode of mobility appeal to a designer, the implementation has a great deal of room for adjustments and options to ensure stability. In this case, we are placing the prismatic joint on vertex $\mathrm{B}$, allowing the edge $\mathrm{AB}$ to move through it, changing the distance between vertices $A$ and B. Again, in the application of this mode of mobility, we must be sure to account for the contact points in order to produce a directional motion. Given perfectly ideal, unidirectional surface contacts, we expect vertex B to remain stationary during the first half of the gait, while vertex A slides towards it. Similarly, in the second half of the gait, vertex A remains stationary while vertex B slides forward (Fig. 7b). The resultant CoM motion resides entirely in the $\mathrm{X}-\mathrm{Z}$ plane (Fig. 8). In application, this mobility mode is very similar to the inchworm motion as mentioned by 


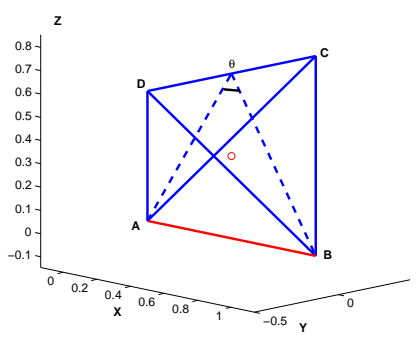

(a)

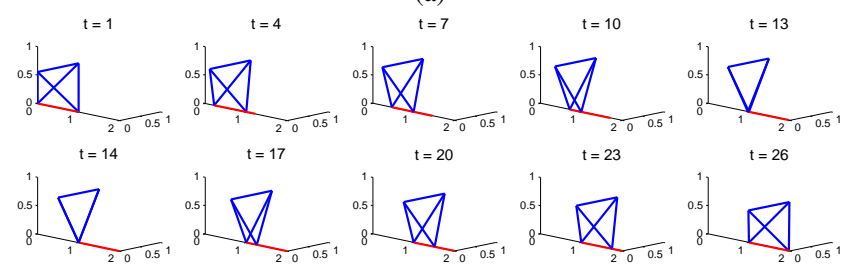

(b)

FIGURE 7: (a)THE TETRAHEDRON BODY IN SUBMODE 1C. THE VERTICES ARE AS FOLLOWS: A $(0,0,0), \mathrm{B}(1,0,0), \mathrm{C}\left(\frac{1}{2}, \frac{1}{2}, \frac{\sqrt{2}}{2}\right), \mathrm{D}\left(\frac{1}{2},-\frac{1}{2}, \frac{\sqrt{2}}{2}\right)$. THE CHOSEN ACTUATION POINT IS $\theta$ (b)THE MOTION OF THE TETRAHEDRON BODY AS IT ACTUATES THE EDGE AB.
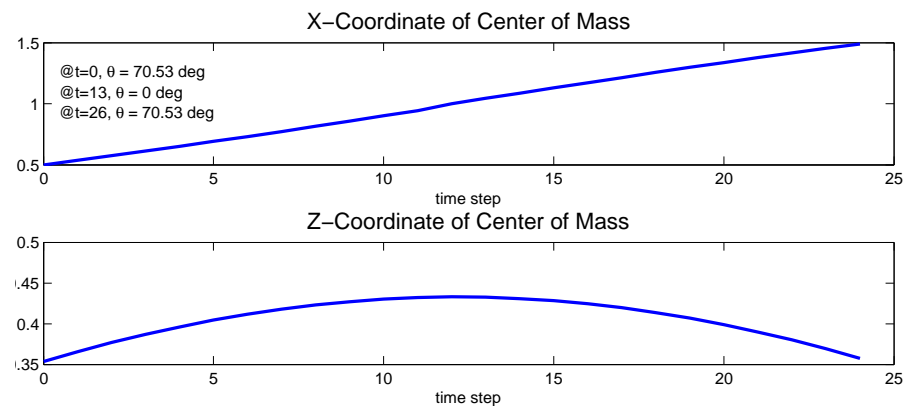

FIGURE 8: SUBMODE 1C: RESULTANT MOVEMENT OF THE COM

Kim [29]. The total travel of the CoM in one gait cycle is 1 edge length.

\section{MOBILITY MODE 2: SINGLE ROTATIONAL DOF}

The idea behind this mode of mobility is to change the distance between two vertices, similarly to Mode 1 , in a different way. Instead of allowing the end of one edge to slide through a prismatic joint, we will give the tetrahedron an additional degree of freedom by placing a hinge in the center of one edge. Recall that we are allowing free rotation at each of the vertices, letting each edge segment rotate in relation to the others. The resultant motion is also very similar to that of Mode 1.

\section{Submode 2A}

Starting with the same initial orientation as in Fig. 2, and using $\theta_{1}$ as indicated in Fig. $2 \mathrm{~b}$ for actuation, the resultant motion can be seen in Fig. 9. The motion of the CoM is identical to that of Mode 1A (Fig. 4). The only additional benefit we gain from using this mode is that we now have an additional vertex point, E. Although it is not one of the original four, and therefore considered massless for our purposes, it can be beneficial as an

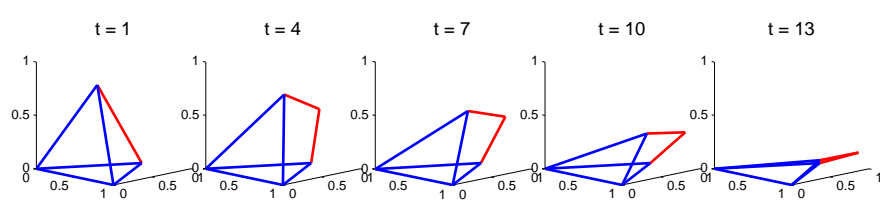

FIGURE 9: MOTION OF THE TETRAHEDRON BODY AS A RESULT OF ADDING A HINGE TO EDGE CD

end-effector for many robotics applications. The range of motion of point $\mathrm{E}$ is shown in Fig. 10.
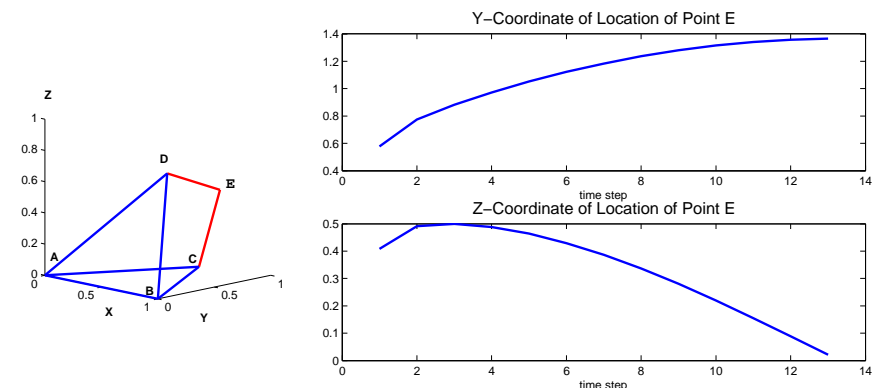

FIGURE 10: ACTUATION OF POINT E AS A RESULT OF ADDING A HINGE TO EDGE CD. THE RANGE OF MOTION IS FROM $\theta_{1}=70.53$ DEGREES TO $\theta_{1}=0$ DEGREES

Finally, in application, this mode boils down to a limited case of a 4-bar mechanism.

\section{Submode 2B}

Following the orientation scheme from Mode 1, we now examine the effects of actuating a "base" edge instead of a side edge. Again, this mode is very similar to the behavior of Mode 1B. Using the same initial orientation and actuation point as shown in Fig. 5a, the resultant motion can be seen in Fig. 11.

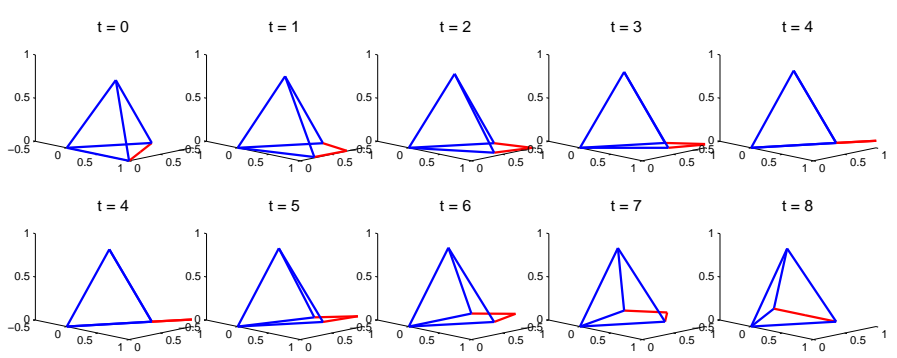

FIGURE 11: MOTION OF THE TETRAHEDRON BODY AS A RESULT OF ADDING A HINGE TO EDGE BC

The resultant motion of the CoM is identical to that of mobility Mode 1B, see Fig. 6. The range of motion of the new point $\mathrm{E}$ is shown in Fig. 12. This mode is a little more different from Mode 2A than Mode 1B is from Mode 1A. That is because with a hinge, we have to worry about where the edge must go to get out of the path of the moving vertices. While Mode 1 simply pushes the extra length linearly outward, Mode $2 \mathrm{~A}$ protrudes point E radially outward while Mode $2 \mathrm{~B}$, obstructed by the "ground," protrudes its point $\mathrm{E}$ in the $\mathrm{X}-\mathrm{Y}$ plane. 

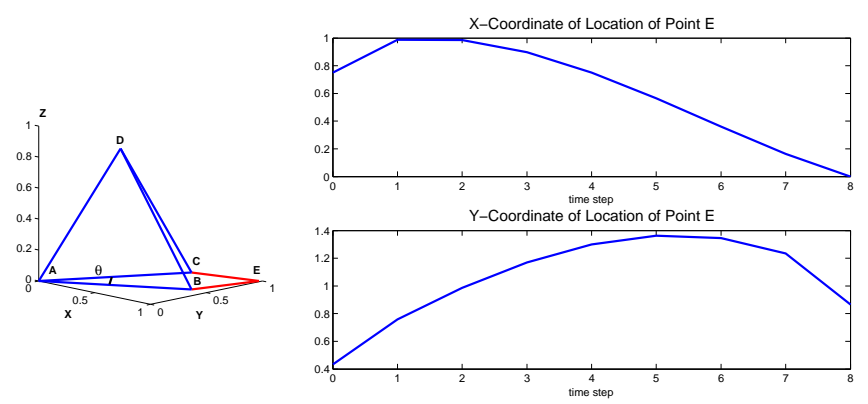

FIGURE 12: ACTUATION OF POINT E AS A RESULT OF ADDING A HINGE TO EDGE CD. THE RANGE OF MOTION IS FROM $\theta=60^{\circ}$ TO $\theta=0^{\circ}$

\section{Submode 2C}

Similarly to $2 \mathrm{~B}$, this submode/mobility mode is analogous to Mode $1 \mathrm{C}$. Like 2B, this mode runs into the issue of where the protruding edge should go. For the sake of slightly simpler equations, let us allow the new point $\mathrm{E}$, the hinge between vertices $\mathrm{A}$ and $\mathrm{B}$, to protrude in the positive $\mathrm{Y}$ direction, in the $\mathrm{X}-\mathrm{Y}$ plane. Using the same actuation point as in Fig. 7a, the resultant motion of the CoM is identical to that shown in Fig. 8. The subsequent motion of the hinge point $\mathrm{E}$ is as shown in Fig. 13. Again, this

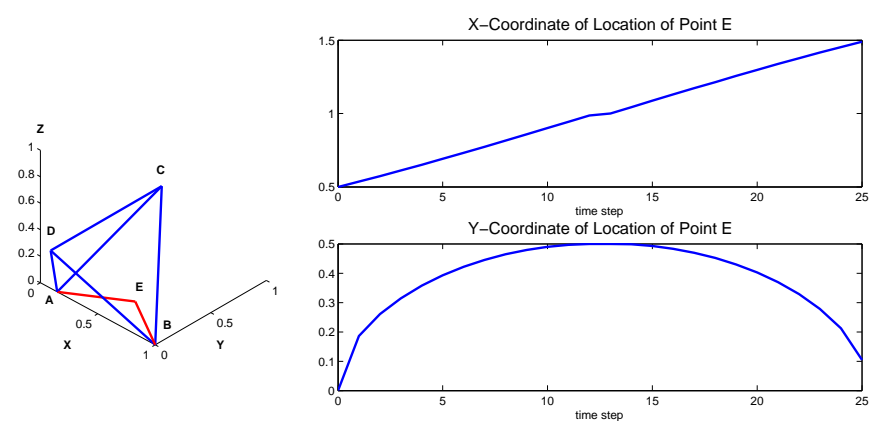

FIGURE 13: ACTUATION OF POINT E AS A RESULT OF ADDING A HINGE TO EDGE AB. THE RANGE OF MOTION IS FROM $\theta=70.53^{\circ}$ TO $\theta=0^{\circ}$ AT TIME $=13$ BACK TO $\theta=70.53^{\circ}$

mechanism can be used to achieve the inchworm motion with a gait cycle travel of 1 .

\section{MOBILITY MODE 3: DOUBLE ROTATION DOF}

Although the modes described in the previous section do not appear significantly different from those in Mobility Mode 1 , they do give rise to a whole new series of available motions. Consider the structures in the previous section. If each of the deformed/hinged edges were allowed to rotate in the axis of their two endpoint vertices, they would be able to achieve motion in a direction perpendicular to those of Mobility Mode 1.

\section{Submode 3A}

As in the previous two sections, this submode is almost a trivial case. However, this is a good demonstration to explain the second degree of rotational freedom. By definition, the additional degree of freedom requires an additional control point. The initial distance between vertices $C$ and $D$ are set in the same way as with Mode $1 \mathrm{~A}$, using $\theta_{1}$ as shown in Fig. 2b. In addition,

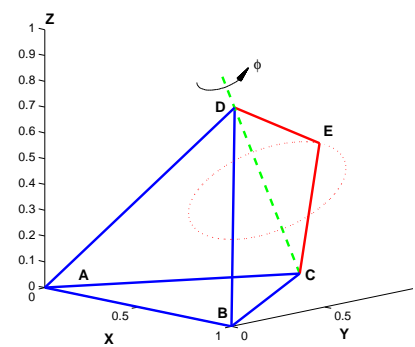

FIGURE 14: ACTUATION OF THE SECOND ROTATIONAL DEGREE OF FREEDOM AS APPLIED TO EDGE CD

we need $\phi$ as shown in Fig. 14 in order to control the rotation of the point $\mathrm{E}$. The benefit of this mode is that we now have out-of-plane motion with point $\mathrm{E}$. In addition, because the circle that $\mathrm{E}$ describes is coupled to our original actuation point $\theta_{1}$, we have also gained the benefit of being able to control the radius of the circle. In application, this will also enable the control of the torque and moment arm about the $\mathrm{CD}$ axis. Within the scope of this study, under the condition that $\mathrm{E}$ and the edge it resides on are massless, the movement of the CoM, regardless of the value of $\phi$ is identical to that of Mobility Mode 1A, as seen in Fig. 4.

\section{Submode 3B}

In comparison with the previous Mobility Mode, this submode is far more interesting. First, let us reorient the original shape as in Fig. 15a. Our edge of interest is BC. As in Fig. 5a,

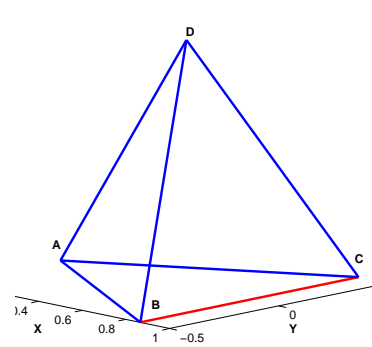

(a)

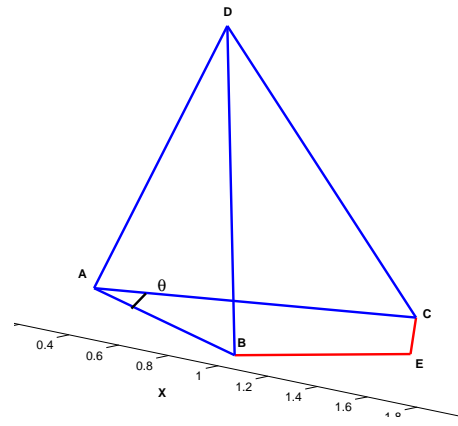

(b)
FIGURE 15: (a)NEW ORIENTATION OF TETRAHEDRON BODY WITH A $(0,0,0)$, $\mathrm{B}\left(\frac{\sqrt{3}}{2}, \frac{1}{2}, 0\right), \mathrm{C}\left(\frac{\sqrt{3}}{2},-\frac{1}{2}, 0\right), \mathrm{D}\left(\frac{\sqrt{3}}{3}, 0, \frac{\sqrt{6}}{3}\right)$. (b)TETRAHEDRON WITH A SLIGHT DEFORMATION IN $\theta$ CAUSING PROTRUSION OF HINGE POINT E

we will control the distance between vertices $B$ and $C$ with the base angle $\theta$ between edges $\mathrm{AB}$ and $\mathrm{AC}$. The result of a slight deformation is shown in Fig. $15 \mathrm{~b}$.

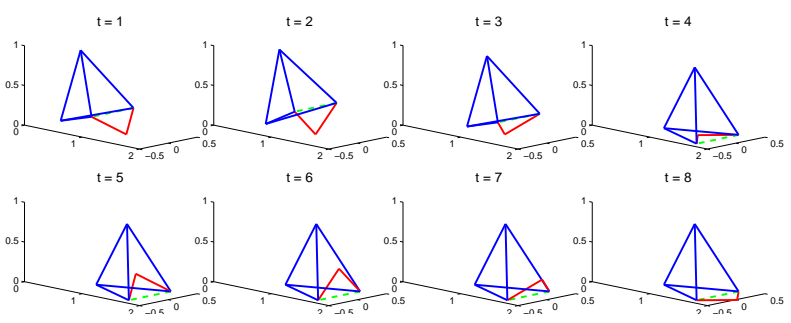

FIGURE 16: PRE-DEFORMED TETRAHEDRON ACTUATING POINT E AROUND AXIS BC 
In this submode, because of the location of the "ground," the vertices $\mathrm{B}$ and $\mathrm{C}$ will lift up off the ground as point $\mathrm{E}$ is rotated around axis $\mathrm{BC}$, maintaining contact only at vertex $\mathrm{A}$, as shown in Fig. 16. To allow this movement, we assume that point E maintains perfectly static contact with the "ground" while it is touching it. We are also choosing to actuate the rotation of the hinged member in one direction only (Fig. 17b). If we had chosen similar conditions to Mobility Mode 1C, i.e. allow both vertex $A$ and point $E$ to be unidirectional contacts, and actuated point $\mathrm{E}$ back and forth in only the lower semicircle, we would once again achieve a form of inchworm motion. In the current form, the hinged member acts more like a wheel, dragging the rest of the tetrahedron forward with each rotation. Due to the vertical component of the motion, the movement of the CoM (see Fig. 17a) is quite different from all the previous cases.

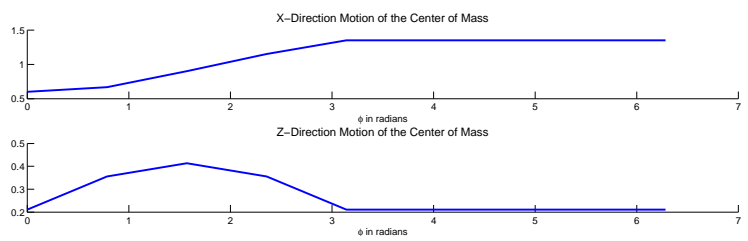

(a)

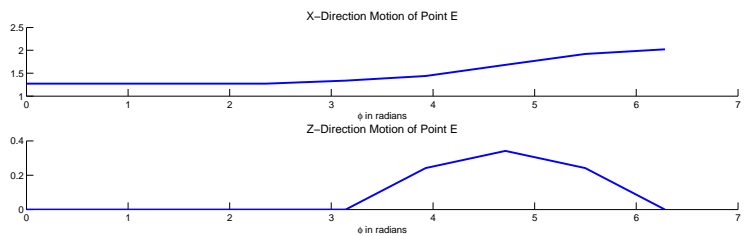

(b)

FIGURE 17: (a)MOTION OF THE COM OF THE TETRAHEDRON BODY AS A FUNCTION OF $\phi, Y=0$. (b)MOTION OF POINT E AS A FUNCTION OF $\phi, Y=0$

This case is particularly interesting because the structure behaves very much like a wheel, a common mode of mobility that we have not yet discussed. In application, this mobility mode corresponds to the extreme case of a Wheg ${ }^{\mathrm{TM}}$ with only one spoke. Similar to another implementation of spoked wheel, the IMPASS [30], the leg length, or in our case radius of rotation, can be changed. This mobility mode is also interesting because, given a mechanism with a large Poisson's Ratio, like an umbrella, we would be able to construct a variable-radius wheel. The travel of the CoM corresponding to one gait cycle is $2 r$ where $r$ is the effective radius of $\mathrm{E}$ due to the $\theta$ deformation.

\section{MOBILITY MODE 4: TWO ADJACENT PRISMATIC EDGES}

The next step in our study is to see what happens when we actuate two edges at the same time. With our unit tetrahedron, there are three distinct ways of choosing two edges. We will start with choosing two non-base edges, then one base, one non-base edge, and finally, two base edges.

\section{Submode 4A}

Starting with the orientation specified in Fig. 15a, allowing edges BD and CD to independently and prismatically actuate through vertex $\mathrm{D}$, we can move vertex $\mathrm{D}$ to anywhere in a section of spherical shell. While the behavior of this mobility mode is not wildly different from that of $1 \mathrm{~A}, 2 \mathrm{~A}$ and $3 \mathrm{~A}$, it does allow us to achieve three dimensional motion with both the location of vertex D and the CoM, as shown in Fig. 18a.

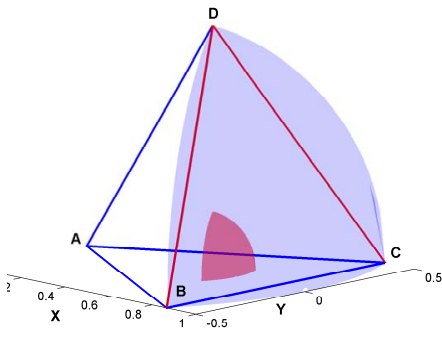

(a)

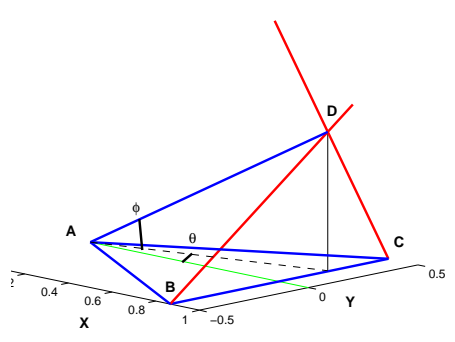

(b)
FIGURE 18: (a)POSSIBLE DEFORMATIONS DUE TO ACTUATION OF EDGES BD AND CD. THE BLUE SURFACE INDICATES THE POSSIBLE LOCATIONS FOR VERTEX D. THE RED SURFACE INDICATES POSSIBLE LOCATIONS OF THE COM. (b)THE LOCATION OF VERTEX D AS ACTUATED BY $\phi$ AND $\theta$

While it is possible to actuate this motion with the control points we have already discussed, perhaps the easiest method for actuating this particular mechanism is by using spherical coordinates. The position of $\mathrm{D}$ can be defined by $\phi$, the angle between the edge $\mathrm{AD}$ and the $\mathrm{X}-\mathrm{Y}$ plane, and $\theta$, the angle of edge $\mathrm{AD}$ around the $\mathrm{Z}$ axis (Fig. 18b). Therefore, as long as vertex $\mathrm{D}$ remains within distance 1 of both vertices $B$ and $C$, it and the CoM can be defined by the following equations:

$$
\begin{gathered}
D=[\cos \phi \cos \theta, \cos \phi \sin \theta, \sin \phi]^{T} \\
C M=\left[\frac{\sqrt{3}}{4}+\frac{\cos \phi \cos \theta}{4}, \frac{\cos \phi \sin \theta}{4}, \frac{\sin \phi}{4}\right]^{T}
\end{gathered}
$$

\section{Submode 4B}

The next adjacent prismatic mobility mode to consider is the combination of actuating one base edge and one non-base edge. This mode is a combination of modes $1 \mathrm{~A}$ and $1 \mathrm{~B}$. It will have all the mobility of the first two mode plus that granted by the interaction of the two. Since we already established that it is possible for the base edge to rotate the location of the CoM around the $\mathrm{Z}$ axis, in this discussion we will use a body-fixed coordinate system to give us a sense of the relative motion. The axes locations are identical to those used in Fig. 18 except that the $\mathrm{X}$ axis will always pass through the midpoint between vertex $\mathrm{B}$ and vertex $\mathrm{C}$. We have also chosen the same relative actuation points as from modes $1 \mathrm{~A}$ and 1B (Fig. 19a).

The range of actuation of $\theta$ is dependent on $\phi$, where $\phi$ : $\left[0, \frac{\pi}{3}\right]$ and $\theta:\left[0, \arccos \left(\frac{\sqrt{3}}{3} \tan \frac{\phi}{2}\right)\right]$. The resultant formulas for vertex $\mathrm{D}$ and the CoM location are as follows.

$$
D=\left[\frac{1}{2 \cos \frac{\phi}{2}}, 0, \frac{\sqrt{3}}{2} \sin \theta\right]^{T}
$$




$$
C M=\left[\begin{array}{l}
\frac{1}{8} \cos \frac{\phi}{2}\left(5+\frac{\sqrt{3} \cos \theta}{\sqrt{1+8 \sin ^{2} \frac{\phi}{2}}}\right) \\
\frac{1}{8} \sin \frac{\phi}{2}\left(\frac{3 \sqrt{3} \cos \theta}{\sqrt{1+8 \sin ^{2} \frac{\phi}{2}}}-1\right) \\
\frac{\sqrt{3}}{8} \sin \theta
\end{array}\right]
$$

Since these calculations are body-fixed, they can be easily translated based on design choices for mechanisms and contacts. The most obvious benefit of this mode from $1 \mathrm{~B}$ is that it allows the structure to change its profile/height. For many applications in an obstructed environment, implementing this mechanism will alleviate some of the movement constraints.

\section{Submode 4C}

The last case of two adjacent, prismatically actuated edges is a combination of two base edges. Once again, we can characterize the mobility exhibited by this mode using a body-fixed coordinate system. In this case we will be using the edge length of $\mathrm{AB}$ and the angle $\theta$ to actuate our motion (Fig. 19b).

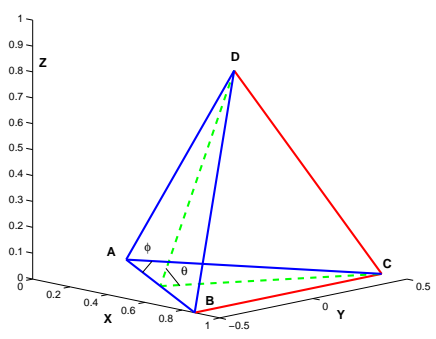

(a)

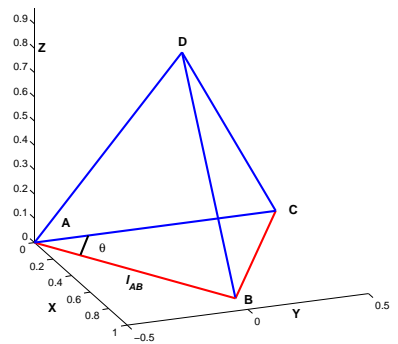

(b)
FIGURE 19: (a)THE ORIENTATION OF MOBILITY MODE 4B AND ITS ACTUATION POINTS $\phi$ AND $\theta$. (b)ORIENTATION OF MOBILITY MODE 4C AND ITS ACTUATION POINTS

The result is, while vertices $\mathrm{A}$ and $\mathrm{C}$ are stationary, vertex $\mathrm{B}$ is actuated in the plane of $z=0$, causing vertex $\mathrm{D}$ to also move. Due to space constraints, we will forego laying out the equations of motion here. By alternating the edge that is actuated, a sort of walking gait can be achieved, as seen in Fig. 20. This demonstrated gait makes the same assumptions about the contact points $\mathrm{A}$ and $\mathrm{C}$ that we did in Mobility Mode $1 \mathrm{~B}$. When edge $\mathrm{AB}$ is actuating, vertex $C$ must maintain perfect static contact with the ground and when edge $\mathrm{BC}$ is actuating, vertex $\mathrm{A}$ must do the same. In this case, our structure is taking off at a 30 degree angle from the $\mathrm{X}$ axis. Of course, in practice, the relative actuation quantities can be adjusted to achieve any planar angle that is required.

\section{MOBILITY MODE 5: TWO OPPOSITE PRISMATIC EDGES}

For completeness of this study, we must examine this last combination of two prismatic edges. There is only one way to
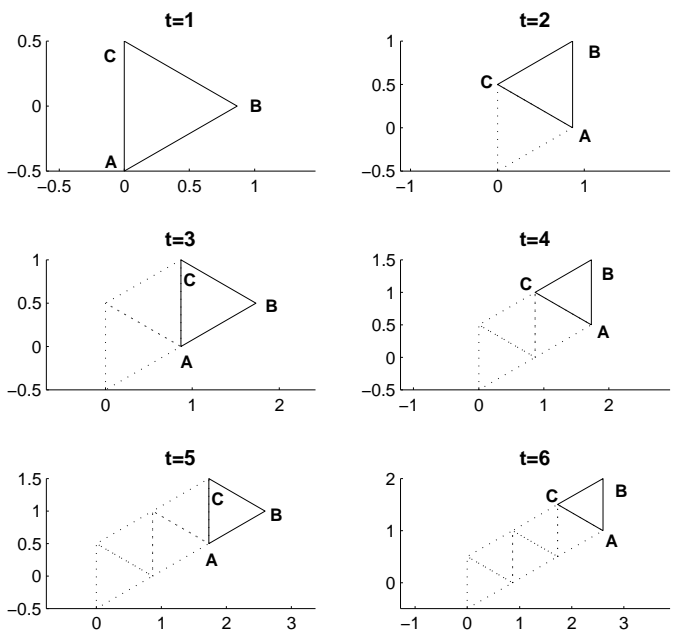

FIGURE 20: PROGRESSION OF THE FOOTPRINT OF THE TETRAHEDRON AS EDGES AB AND BC ARE ALTERNATELY ACTUATED

select two non-adjacent edges on this tetrahedron. One of them must be a base edge and the other must be a non-base edge.

\section{Submode 5A}

For the benefits of symmetry in our numbers, we will once again use the starting orientation from Fig. 15. We will be actuating edges $\mathrm{AD}$ and $\mathrm{BC}$ and the points of actuation were chosen as shown in Fig. 21. The resulting motions for vertex $\mathrm{D}$ and the $\mathrm{CoM}$ are given by:

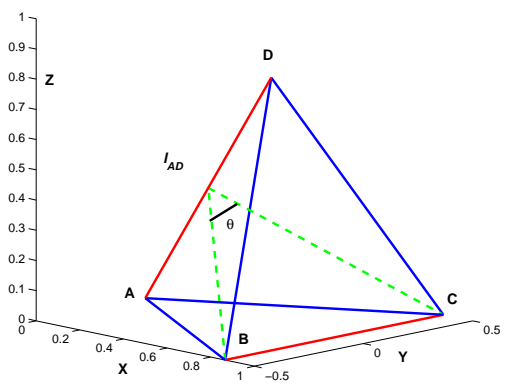

FIGURE 21: THE ORIENTATION OF MODE 5A AND ITS ACTUATION POINTS $l_{A D} \operatorname{AND} \theta$

$$
\begin{gathered}
D=\left[\frac{l_{A D}^{2}}{2 \cos \theta}, 0, \sqrt{l_{A D}^{2}-\frac{l_{A D}^{4}}{4 \cos ^{2} \theta}}\right]^{T} \\
C M=\left[\frac{1}{2} \cos \theta+\frac{l_{A D}^{2}}{8 \cos \theta}, 0, \frac{1}{4} \sqrt{l_{A D}^{2}-\frac{l_{A D}^{4}}{4 \cos ^{2} \theta}}\right]^{T}
\end{gathered}
$$

While the actual behavior of the body is not very different from Mobility Mode 4B, in this orientation, the equations are a little simpler. Once again, being able to actuate one base edge grants us the same motion as in Mobility Mode 1B. Manipulating the 
non-base edge allows us to change the profile of the entire structure. Due to the slightly different relative positions of the actuated edges, the total range of CoM motion is increased to 0.56 .

\section{MOBILITY MODE 6: TWO ADJACENT DOUBLE ROTA- TIONAL EDGES}

We have previously shown that giving one of the edges a single rotational degree of freedom is not very different from making that edge prismatic. It is, in fact, the limiting case of the double rotational degree of freedom, Mode 3. In this section, we will forego the discussion of single rotation edges and jump straight into the double rotational ones. Again, we will follow the scheme of choosing two non-base edges, one base, one non-base edge, and finally, two base edges.

\section{Submode 6A}

For this case, we will be using the same starting orientation as Mobility mode 4A (Fig. 18a) as well as the same actuation points $\theta$ and $\phi$ (Fig. 18b). The main difference is, we will need to define two additional points, $\mathrm{E}$ and $\mathrm{F}$, as the midpoints/hinge locations of edges BD and CD, respectively (Fig. 22a). The result of the deformation and subsequent positioning of vertex D determines the available range of motion for points $\mathrm{E}$ and $\mathrm{F}$. For:

$$
D=[\cos \phi \cos \theta, \cos \phi \sin \theta, \sin \phi]^{T}
$$

$\mathrm{E}$ is then a circle of radius

$$
r_{E}=\frac{1}{2} \sqrt{\sqrt{3} \cos \phi \cos \theta-\cos \phi \sin \theta-1}
$$

around the point

$$
\text { Cent }_{E}=\frac{1}{2}\left[\cos \phi \cos \theta+\frac{\sqrt{3}}{2}, \cos \phi \sin \theta-\frac{1}{2}, \sin \phi\right]^{T}
$$

on the plane

$$
x\left(\cos \phi \cos \theta-\frac{\sqrt{3}}{2}\right)+y\left(\cos \phi \sin \theta+\frac{1}{2}\right)+z \sin \phi=0
$$

The equations for point $\mathrm{F}$ are derived in a similar manner and are not included here due to space constraints. Please see [25] for more details.

While this mobility mode gives us a wider range of motion on the part of both point $\mathrm{E}$ and point $\mathrm{F}$, it also brings up a very troublesome complication, interference between the members. Consider the case where both $\mathrm{E}$ and $\mathrm{F}$ are actuated equally to the maximum extent possible, at $\phi=\theta=0$. Edge AD would be coplanar with the base of the tetrahedron and bisecting edge $\mathrm{BC}$. Aside from the fact that rotating either $\mathrm{E}$ or $\mathrm{F}$ would cause the base of the tetrahedron to lift up off the "ground," a situation we have already dealt with in Mobility Mode 3B, neither E nor F would be able to move in a full cycle because of the presence of the edge BC. As another example, should the radius of E's circle of motion overlap with that of $\mathrm{F}$, there is also the possibility of collision during rotation.

Depending on the specific application, there are many different ways of working around the interference problem. The engineer could stagger the spin of $\mathrm{E}$ and $\mathrm{F}$, limit the range of actuation, or even choose to actuate only one of the two at any given time. These are only three possible methods for mitigating interference. With the proper foresight and exploration, it does not have to be a show-stopper.

\section{Submode 6B}

This submode (Fig. 22b) is a combination of Modes 3A and 3B. Like Mode 3B, the base edge rotation has a direct impact on the location of vertices $\mathrm{B}, \mathrm{C}$ and $\mathrm{D}$ while the non-base edge rotational range of point $\mathrm{F}$ is a result of the final positions.

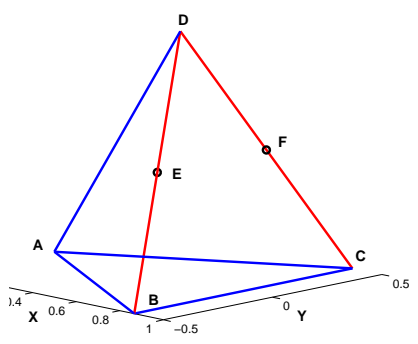

(a)

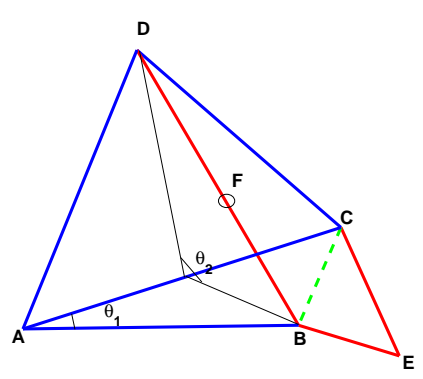

(b)
FIGURE 22: (a)THE ORIENTATION OF MODE 6A AND NEW HINGE POINTS E AND F. (b)THE ORIENTATION OF MODE 6B AND NEW HINGE POINTS E AND F.

Due to the increased degrees of freedom and the interdependency of the vertex and point locations, the equations become much more complicated. Due to spatial constraints, we will not list them here. Interested readers may find them in the technical report [25]. The benefit of this mode is that the body can move, with a variable gait, linearly while changing the shape/size of its profile. In close quarters, it can perhaps also push along a wall or move obstacles with the protrusion of point $\mathrm{F}$.

\section{Submode 6C}

For completeness, it behoves us to discuss the case where both double rotationally actuated edges are on the base. An example of a pre-deformed structure of this Mobility Mode is shown in Fig. 23. The distance between vertices $A$ and $B$ and vertices $B$ and $C$ are actuated by angles $\theta_{1}$ and $\theta_{2}$. Further, the rotation of point $\mathrm{E}$ around axis $\mathrm{AB}$ and point $\mathrm{F}$ around axis $\mathrm{BC}$ should also be actuated. At this point, recall that we have used assumptions such as perfect balance in Mobility Mode 1C and $3 \mathrm{~B}$. Because of the geometry of the current structure, it becomes difficult to decide exactly which set of assumptions should take precedence. If we were to actuate only one edge at a time, we can maintain our assumption of $3 \mathrm{~B}$ that the tetrahedron can and will balance on either vertex $A$ and point $F$ or vertex $C$ and point E. This would result in a walking gait, like a combination of Mobility Mode 3C and 4C. On the other hand, we cannot maintain these assumptions if the structure intends to actuate both edges 


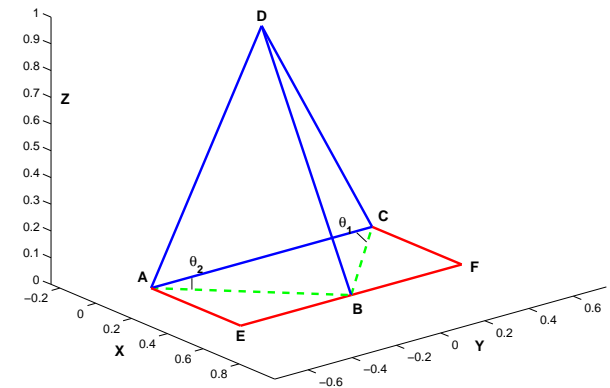

FIGURE 23: THE ORIENTATION OF MODE 6C AND NEW HINGE POINTS E AND F.

at the same time. Actuating point E expects vertex A to lift off the "ground" and actuating point $\mathrm{F}$ expects vertex $\mathrm{C}$ to elevate. Those two things cannot happen concurrently and still maintain the illusion of real world physics. Therefore, should both edges need to actuate at the same time, another analysis would have to be done to determine which contact points would still be touching the "ground." The resultant motion can be likened to a small child sitting on the floor and pulling himself forward using his legs, which may not be extended the same amount. By adjusting the relative extended-ness, he may travel in arcs to either side or move forward linearly.

The equations of motion of this situation are highly dependent on what type of behavior is expected of the structure and we will forego the lengthy derivation for each subcase in this paper.

\section{MOBILITY MODE 7: TWO NON-ADJACENT DOUBLE ROTATIONAL EDGES \\ Submode 7A}

As we mentioned in the discussion of Mobility Mode 5, there is only one way to select two non-adjacent edges. One of them must be a base edge and the other must be a non-base edge (Fig. 21). All of the equations of motion that are required to characterise the behavior of this case have already been covered in previous sections. The truly interesting part of this case is the fact that the two articulated edges are essentially perpendicular and offset wheel axles. In fact, vehicles like this already exist in the toy market, as shown in Fig. 24.

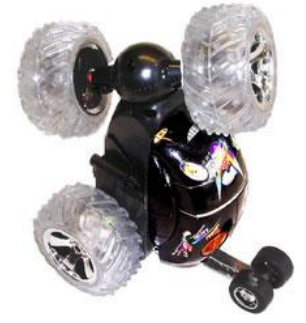

FIGURE 24: STUNT GENIUS REMOTE CONTROLLED CAR

The great benefit of these type of vehicles, like other vehicles where the wheel diameters are the largest dimension of the structure, is that they are incredibly resistant to overturning. This makes them perfect for rough terrains, unstable environments and even being dropped out of a larger vehicle. These mechanisms are able to function in whichever orientation they happen
TABLE 1: SUMMARY OF RESULTS

\begin{tabular}{|c|c|c|c|c|}
\hline 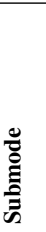 & ֻี & 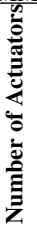 & 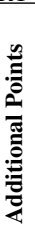 & 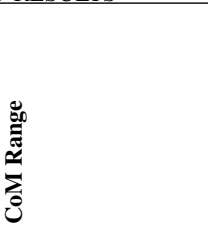 \\
\hline $1 \mathrm{~A}$ & Oscillation & 1 & 0 & 0.25 \\
\hline 1B & Rotational Gait & 1 & 0 & 0.66 \\
\hline $1 \mathrm{C}$ & Inchworm & 1 & 0 & 1 \\
\hline $2 \mathrm{~A}$ & Oscillation & 1 & 1 & 0.25 \\
\hline $2 \mathrm{~B}$ & Rotational Gait & 1 & 1 & 0.66 \\
\hline $2 \mathrm{C}$ & Inchworm & 1 & 1 & 1 \\
\hline $3 \mathrm{~A}$ & $\begin{array}{l}\text { Oscillation; } \\
\text { Rotation }\end{array}$ & 2 & 1 & 0.25 \\
\hline 3B & $\begin{array}{l}\text { Rotation; } \\
\text { Inchworm; } \\
\text { Wheelbarrow }\end{array}$ & 2 & 1 & $2 * r_{E}=\left[0, \frac{1}{2}\right]$ \\
\hline $4 \mathrm{~A}$ & 3D Oscillation & 2 & 0 & 0.25 \\
\hline $4 B$ & $\begin{array}{l}\text { Oscillation; } \\
\text { Rotation }\end{array}$ & 2 & 0 & $0.25 ; 0.66$ \\
\hline $4 \mathrm{C}$ & $\begin{array}{l}\text { Rotational Gait; } \\
\text { Walking }\end{array}$ & 2 & 0 & 1.14 \\
\hline 5 & $\begin{array}{l}\text { Oscillation; } \\
\text { Rotation }\end{array}$ & 2 & 0 & 0.56 \\
\hline $6 \mathrm{~A}$ & 3D Oscillation & 4 & 2 & 0.25 \\
\hline $6 \mathrm{~B}$ & $\begin{array}{l}\text { Oscillation; } \\
\text { Inchworm; } \\
\text { Wheelbarrow }\end{array}$ & 4 & 2 & $0.25 ; 2 * r_{E}=\left[0, \frac{1}{2}\right]$ \\
\hline $6 \mathrm{C}$ & $\begin{array}{l}\text { Inchworm; } \\
\text { Wheelbarrow } \\
\text { Walking }\end{array}$ & 4 & 2 & Highly Variable \\
\hline 7 & $\begin{array}{l}\text { Oscillation; } \\
\text { Inchworm; } \\
\text { Wheelbarrow }\end{array}$ & 4 & 2 & $2 * r_{E}=\left[0, \frac{1}{2}\right]$ \\
\hline
\end{tabular}

to be in or are able to right themselves into the proper orientation for performing their functions.

\section{CONCLUSION}

In this paper, we have extensively explored the modes of mobility that arise from slight modifications of an edge-only unit tetrahedron. Table 1 shows a summary of all the different modes we have discussed. As one can see, there are many overlapping areas within the different modes. In addition, as we mentioned at the beginning, there are many ways of modifying and customizing the different modes for specific applications. The mobility modes covered in this paper should only serve as a starting point for design.

The next step in this effort is to deconstruct our tetrahedron even further in order to try and capture more modes of mobility. One of the topics we have not yet explored is tread motion. How many additional degrees of freedom must be added before we can achieve that with our starting shape? What about the side-winder snake motion that has been explored by many other robotics engineers in the past?

Concurrently, we will also examine the possibility of building a model of this tetrahedron, incorporating all the degrees of freedom we have discussed in this paper. Building and actuating 
a real model of this body will provide better understanding of how reality, with all its imperfections of heat, friction, and other inefficiencies compare to the mathematically derived simulation. Having actual hardware will also allow us to drive the structure in a open loop to test its limitations and find its constraints.

\section{ACKNOWLEDGMENT}

The Authors would like to thank the US ARMY, ARDEC Picatinny Arsenal for their support in funding this research through the ARDEC Science Fellowship.

\section{REFERENCES}

[1] Groover, M., Weiss, M., Nagel, R., and Odrey, N., 1986. Industrial Robotics, Technology, Programming, and Applications. McGraw-Hill, New York, NY.

[2] Beckar, M., 1969. Introduction to Terrain-Based Vehicle Systems. University of Michigan Press, Ann Arbor, MI.

[3] Hirose, S., 1984. "A study of design and control of a quadruped walking vehicle". The International Journal of Robotics Research, 3(2), June, pp. 113-133.

[4] Song, S., and Waldron, K., 1987. "Geometric design of a walking machine for optimal mobility". Journal of Mechanisms, Transmissions, and Automation in Design, 109(1), pp. 21-28.

[5] Byrd, J., and DeVries, K., 1990. "A six-legged telerobot for nuclear applications development". The International Journal of Robotics Research, 9(2), April, pp. 43-52.

[6] McGeer, T., 1990. "Passive dynamic walking". The International Journal of Robotics Research, 9(2), April, pp. 6282.

[7] Simmons, R., and Krotkov, E. "An integrated walking system for the ambler planetary rover". Proceedings of the 1991 IEEE International Conference on Robotics and Automation, 3.

[8] Iagolnitzer, M., Richard, F., and Samson, J. "Locomotion of an all-terrain mobile robot". Proceedings of the 1992 IEEE International Conference on Robotics and Automation, 1.

[9] Burdick, J., and J. Radford, G. C. "A 'sidewinding' locomotion gait for hyper-redundant robots". Proceedings of the 1993 IEEE International Conference on Robotics and Automation, 3.

[10] Full, R., Earls, K., Wong, M., and Caldwell, R., 1993. "Locomotion like a wheel?". Nature, 365(7), October, p. 495.

[11] Yim, M. "A reconfigurable modular robot with many modes of locomotion". Proceedings of JSME International Conference on Advanced Mechatronics.

[12] Kinnear, K., ed. Advances in Genetic Programming.

[13] Ebefors, T., Mattson, J., Kalvesten, E., and Stemme, G. “A walking silicon micro-robot". The 10th Int Conference on Solid-State Sensors and Actuators (Transducers99).

[14] Choset, H., Lynch, K., Hutchinson, S., Kantor, G., Burgard, W., Kavraki, L., and Thrun, S., 2005. Principles of Robotic Motion: Theory, Algorithms, and Implementations. MIT Press, Cambridge, MA.

[15] Ingram, M., and Ingram, M., 2005. "Whole skin locomo- tion inspired by amoeboid motility mechanisms". In 29th ASME Mechanisms and Robotics Conference.

[16] Hong, D. "Biologically inspired locomotion strategies: Novel ground mobile robots at romela". The $3^{\text {rd }} A n$ nual Conference on Ubiquitous Robots and Ambient Intelligence.

[17] Sahai, R., Avadhanula, S., Groff, R., Steltz, E., Wood, R., and Fearing, R. "Towards a $3 \mathrm{~g}$ crawling robot through the integration of microrobot technologies". IEEE International Conference on Robotics and Automation.

[18] Driesen, W., Rida, A., Breguet, J., and Clavel, R. "Friction based locomotion module for mobile mems robots". IEEE/RSJ International Conference on Intelligent Robots and Systems.

[19] Wood, R., 2008. "The first takeoff of a biologically inspired at-scale robotic insect". IEEE Transactions on Robotics, 24(2), April, pp. 341-347.

[20] Morazzani, I., Hong, D., Lahr, D., and Ren, P. "Novel tripedal mobile robot and considerations for gait planning strategies based on kinematics". Recent Progress in Robotics, LNCIS, 370.

[21] Ho, T., Choi, S., and Lee, S. "Development of a meso-scale self-contained bounding robot". Proceedings of the 2008 International Conference on Robotics and Biomimetics.

[22] Volpe, R., and Balaram, J., 1996. "Rocky 7: a next generation mars rover prototype". Advanced Robotics, 11(4), pp. 341-358.

[23] Yu, J., Wang, L., and Tan, M. "A framework for biomimetic robot fish's design and its realization". Proceedings of the American Control Conference, 3.

[24] Nair, R., and Ito, T. "Robocup rescue: A proposal and preliminary experiences". $4^{\text {th }}$ Annual International Conference on Multi-Agent Systems.

[25] Chen, Y., and Cappelleri, D., 2012. Mobility modes arising fron the breakdown of a basic regular tetrahedron. Technical Report 1, Stevens Institute of Technology, Hoboken, NJ, Feburary. URL https://sites.google.com/site/... multiscaleroboticsautomation/... SITTechReportFeb2012.pdf.

[26] Hu, B., and Wang, L., 2009. "A miniature wall climbing robot with biomechanical suction cups". Industrial Robot: An International Journal, 36(6), pp. 551-561.

[27] Kim, S., Spenko, M., Trujillo, S., Heyneman, B., Santos, D., and Cutkosky, M., 2008. "Smooth vertical surface climbing with directional adhesion". IEEE Transactions on Robotics, 24(1), Feburary, pp. 65-74.

[28] Daltorio, K., and Gorb, S. "A robot that climbs walls using micro-structured polymer feet". Climbing and Walking Robots, 3.

[29] Kim, S., and Kim, S., 1999. "Precise rotary motor by inchworm motion using dual wrap belts". Review of Scientific Instruments, 70(5), pp. 2546-2550.

[30] Hong, D., and Laney, D. "Preliminary design and kinematic analysis of a mobility platform with two actuated spoked wheels". Proceedings of the 2005 IEEE/RSJ Conference on Intelligent Robots and Systems. 